\title{
Rationalizing viral drug target identification using computational approaches : The SARS-CoV-2 Spike Glycoprotein S Case Study
}

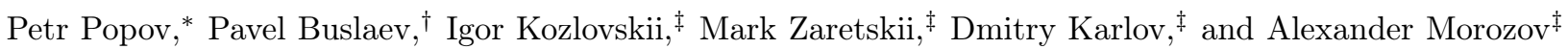

\section{ABSTRACT}

COVID-19 emphasized the need for fast reaction tools to fight global biological threats such as viruses. Rapid drug discovery is one of the strategies for efficient social response. The success of a drug discovery campaign critically depends on the selected drug target, and the wrong target nullifies all the efforts to develop a drug. Viral drug target identification is a challenging problem, and computational methods can reduce the number of candidate targets. Here we present a structurebased approach to identify vulnerable regions in viral proteins that comprise drug binding sites. To detect promising binding sites, we take into account protein dynamics, accessibility, and mutability of the binding site, coupled with the putative mechanism of action of a drug. Applying to the SARS-CoV-2 Spike Glycoprotein S, we observed conformation- and oligomer-specific glycan-free binding site that is proximal to the receptor binding domain and comprises topologically important amino acid residues. Molecular dynamics simulations of Spike in complex with drug-like molecules docked into the binding sites revealed shifted equilibrium towards the inactive conformation compared to the ligand-free simulations. Small molecules targeting this binding site could prevent the closed-to-open conformational transition of the Spike protein, thus, allosterically inhibit the interaction with the human angiotensin-converting enzyme 2 receptor.

\section{INTRODUCTION}

The COVID-19 pandemic, started in December 2019, caused over a million human deaths worldwide and became a global challenge of the 21st century. Although the closely related coronaviruses SARS-CoV and MERS had been known and studied for over a decade, humankind turned out to be helpless against a novel strain, SARS$\mathrm{CoV}-2$. The World Health Organization reported little or no therapeutic effect for one of the most promising anticovid drugs: remdesivir, hydroxychloroquine, lopinavir, and interferon 33. Unprecedented scientific collaborative efforts are being made to develop antiviral therapies,

\footnotetext{
* iMolecule, CDISE, Skolkovo Institute of Science and Technology, Moscow, Russia; p.popov@skoltech.ru

$\dagger$ Nanoscience Center and Department of Chemistry, University of Jyväskylä, Finland

¥ iMolecule, CDISE, Skolkovo Institute of Science and Technology, Moscow, Russia
}

such as vaccines and drug-based treatments, emphasizing the need for fast and efficient reaction tools to fight viruses on the molecular level. Computational structurebased drug design approaches are matured to high-level precision and take a relatively short time to be applied for a drug target of interest [4]. One of the most common drug target types is proteins; a drug binds to a spatial region in the protein structure, called the binding site [32. However, target and binding site identification is not straightforward and can be arguably considered as one of the most challenging and critical parts of the drug discovery campaign [3, 37. Typical obstacles in binding site identification include pitfalls related to the i) flexibility, ii) druggability, iii) accessibility and iv) mutability of a protein. Firstly, protein flexibility is crucial in drug discovery 42, and a binding site may be present or absent in a given three-dimensional structure; hence, there is a risk of overlooking a relevant binding site or detecting a fleeting irrelevant binding site [22]. Secondly, not every detected binding site is 'druggable', meaning that one can make a drug that modulates protein function upon binding [3]. Thirdly, the binding site must be accessible to a potential drug; for example, viral proteins can be glycosylated hence shielding its surface from drug binding [47. Fourthly, viral proteins are adaptable to amino acid substitutions; therefore, a binding site in one viral strain can be modified or ruined in another strain [54]. Another essential concern is that for newly discovered binding sites, typically, there is a lack of understanding of the modulation mechanism of a potential drug molecule, that limits drug design against novel viral strains or for personalized medicine purposes 38. Computational approaches that consider the issues mentioned above would help reduce the high rate of false-positive drug target binding sites [8, 23] and facilitate a faster social response in case of future pandemics.

Here we rationalize viral target identification by considering flexibility, druggability, accessibility, and mutability of the protein target, as well as the putative mechanism of action of a potential drug. We used Spike Glycoprotein S (Spike) as the protein target that covers spherically-shaped SARS-CoV-2 virions [46]. Spike has homotrimeric architecture and consists of three subunits responsible for binding to the host cell and merging cellular-viral membranes [52, [57]. A subunit contains the receptor-binding domain (RBD) that undergoes large confromational transitions from the closed (down RBD conformation, PDB: 6VXX [46]) to the open (up RBD conformation, PDB: 6VSB [51]) Spike states. In the open conformation, the virus is capable of binding to the peptidase domain (PD) of angiotensin-converting 
enzyme 2 (ACE2) with one of its subunit RBDs followed by the fusion process [40, 53]. One of the strategies to prevent viral infection is to design a protein-protein interaction inhibitor that directly blocks the RBD-PD interaction interface [4, 11. However, such inhibitors could be challenging to design because of highly glycosylated Spike and ACE2 [47, 56]. Another concern is that RBD comprises highly variable amino acid residues, potentially making identified blockers ineffective against different viral strains [10. Finally, direct RBD-PD inhibitors may affect normal ACE2 function leading to side effects upon binding to it. Therefore, drugs targeting more accessible and conservative regions in the Spike structure would be safer and have broader applicability, as compared to the direct RBD-PD inhibitors; a lot of experimental and computational efforts are being made to describe such a distinct region ([6, 7, 17, 19, 20, 24, 41, 54, 58, to name a few).

In this study, we identified a vulnerable region in the Spike trimer structure that could be used to allosterically inhibit RBD-PD interactions by preventing closed to open conformational transition of Spike. We analyzed the long-range molecular dynamics (MD) trajectories of Spike using a spatiotemporal deep learning-based approach BiteNet 22] and selected conformation- and oligomer-specific cryptic binding site based on the putative mechanism of action and structure-based criteria. Namely, the detected binding site is formed by two spike subunits, it shares amino acid residues with the RBD of one subunit, and it is present in the closed, but not the open conformation of Spike. The binding site is accessible for small molecules and free from glycans. We applied sequence-based and structure-based network analysis to show that amino acid residues comprising the binding site are more conservative and less tolerant of mutations, as compared to RBD, indicating a broader application of potential drugs targeting this binding site against viruses from the Coronaviridae family. We analyzed the "druggability" of the detected binding site by molecular docking of the drug-like chemical library and revealed several high-scored compounds that fit the binding site. We further compared the flexibility of ligand-free and ligand-bound Spike conformations using MD simulations and identified four drug-like molecules that stabilize Spike in the closed conformation; the four molecules can be used as the starting points for anticovid drug design. Therefore we hypothesize that ligands bound to the detected binding site could lock Spike in the closed state conformation, preventing viral association with the host cell. Overall, the presented computational approach could help identify the most relevant viral drug target binding sites for drug discovery and design.

\section{RESULTS AND DISCUSSION}

To allosterically inhibit the RBD-PD interactions, we looked for a vulnerable region in the Spike structure in- volved in the conformation transition from the closed to the open state. Such a region can be exploited for drug discovery to disrupt the conformational transition, hence, inhibiting viral activity. To locate a binding site in the Spike trimer structure that could be used to lock it in the closed state conformation, we searched for a spatial region that:

- involves two or three subunits of the spike (oligomer-specific)

- is observed in the closed but not in the open state conformation (conformation-specific)

- is located in the proximity of RBD

- can fit drug-like molecules

- is not sheltered with the glycan molecules

- is less prone to mutations

The first three criteria aim to select a region involved in the RBD movement; the fourth and fifth criteria assure that this region can be exploited for drug discovery; and the last criterion aims to expand the applicability of potential drugs across the different viral strains.

To detect binding site satisfying those rationales, we applied BiteNet [22], a deep learning approach for spatiotemporal identification of druggable binding sites, to the $10 \mu \mathrm{s}$ MD simulation trajectories by D.E. Shaw Research for the Spike structure in the closed and prefusion states [17] (see VA). Out of 202 putative predictions, 51 passed the probability score thresholds; of these 30 binding sites comprise amino acid residues from the two or three Spike subunits. This is followed by the conformation-specific filter that kept 16 putative binding sites present in the closed, but not in the pre-fusion state conformation (see Figure 1 b). The subsequent filter left seven putative binding sites with the median topological importance higher than one calculated for Spike (see $\widehat{\mathrm{VC}}$ ). Among these, three are located in the regions occupied by glycans, thus, yielding four pre-final candidates. Taking into account the proximity of the RBD domain left us with only two remaining binding sites. Finally, along the $10 \mu \mathrm{s}$ MD trajectory, one binding site was present in $52.5 \%$ of frames (2630 out of 5005 ), while the other only in $5 \%$ (250 out of 5005) (see Figure 1 a,c).

The most promising detected binding site is formed by the two neighboring subunits and comprises amino acid residues from $\mathrm{RBD}$ of one subunit (see Figure 1 $\mathrm{k}$ ). We observed such a binding site for each pair of interacting subunits, and only one binding site, that corresponds to RBD in the up conformation, is collapsed in the prefusion state. We want to note that the BiteNet probability score for this binding site varies from 0.0 to 0.7 across the MD trajectory (see Figure 1 d); therefore, it can be overlooked in a static structure of Spike. On the other hand, this binding site is continuously observed along the MD trajectory, indicating that it is indeed a binding site, rather than a fleeting prediction. These emphasizes role 
of MD trajectory analysis in the binding site identification.

To analyze the "druggability" of the detected binding site, we used molecular docking to screen $\sim 8,000 \mathrm{FDA}$ approved, experimental and investigational compounds retrieved from the DrugBank database [49] using the conformation corresponding to the highest probability score of the binding site (see VD). We observed $\sim 200$ druglike molecules that nicely fit into the binding site with the high docking scores (Score $\leq-30.0$ ) and form interactions with both subunits (see Figure2).

The detected binding site can fit compounds of different molecular weight, octanol-water partition coefficient, topological polar surface area, number of hydrogen bond donors and acceptors, and number of rotatable bonds (see Figure 2a,b). High score values for some of the compounds, however, might be an artifact of molecular docking given a large number of possible polar contacts (for example, see Figure 2b, CID: 193491). We, therefore, selected 20 drug-like molecules for further investigation from the hit list, excluding potential artifacts, as well as highly similar compounds (see Supplementary Table 2); Figure 2 $\mathrm{c}$ shows superimposed docking poses of these compounds along with the BiteNet predicted binding site.

We hypothesize that such small molecules can stabilize bridges, preventing the closed-to-open conformational transition of Spike, thus, abolishing viral activity. To support this hypothesis, we run 100 ns MD simulations of the ligand-bound Spike structures for the 20 selected compounds. Given that the trimer structure is asymmetric and that only one Spike subunit undergoes the closed-to-open conformational transition, we used three compounds placed to three binding sites formed by $A-C$, $C-B$, and $B-A$ subunits. Next, we analyzed RBD's flexibility in terms of the root-mean-squared-deviation (RMSD) and compared it to the ligand-free MD simulations. We observed that for four out of 20 compounds, the maximal $\mathrm{RBD}$ deviation is almost twice lower, as compared to the ligand-free simulations (see Figure 3a,b). More precisely, RBD of one of the subunits deviates for $\sim 10.0 \AA$ by the end of the ligandfree simulation, while the RMSD values for each RBD in ligand-bound simulations do not exceed $\sim 5.0 \AA$ (see Figure $3 \mathrm{c}$ ). Supplementary Figure 1 shows RMSD values for the RBD domain and the protein-ligand interaction interface for all 20 compounds. For the rest 16 compounds, we observed at least one pair of subunits with similar RMSD values compared to the ligand-free simulation.

It is important to note that $100 \mathrm{~ns}$ simulations are not enough to observe the RBD transitions from the closed to the fully open state, and longer simulations are required to capture this event [58]. Consequently, the $16 \mathrm{com}-$ pounds might have an inhibition effect beyond the $10 \AA$ RBD deviation, but we did not investigate this because of computational limitations. However, we did observe the difference between the ligand-bound and ligand-free sim- ulations in terms of the the RBD deviation on a smaller scale for four drug-like molecules.

Analysis of the protein-ligand interactions revealed 27 amino acid residues within $4 \AA$ from the ligands across the MD trajectories. To evaluate the flexibility of these 27 amino acid residues, we calculated the root-meansquared-fluctuations (RMSFs) across the MD trajectories, and Figure 4 shows the obtained RMSF profiles for the ligand-bound and ligand-free simulations. We observed that the amino acid residues are more stable on average, especially the K462-P463-F464-E465 region of the RBD domain, where the RMSF value is at most $2 \AA$ for the ligand-bound trajectories. Among these residues, we observed eight that form dense contacts maintaining during the simulations, namely Y200 and P230 of one subunit and Y396, D428, F429, F464, S514, and E516 by the other subunit (see Figure $4 \mathbf{a}$ ). The ligands form hydrophobic contacts with Y200 and P230 of one subunit, Y200 also form $\pi$-stacking interactions and hydrogen bonds via its phenolic oxygen. For the other subunit, one can note hydrophobic contacts formed with Y396 and F454, as well as $\pi$ - and T-stacking interactions, and hydrogen bonds formed by E516 with the ligands. Figure $4 \mathrm{c}$ shows the percentage of time these amino acid residues were within $4.0 \AA$ from the ligand for each interaction interface during the molecular dynamics simulation.

Variability of the amino acid sequence is one of the biggest challenges in drug re-purposing and drug discovery against viruses. As a consequence, drugs targeting less variable binding regions might be effective across the different viral stamps. It was shown that the topological importance of the amino acid residues is critical in accessing mutational tolerance for viral proteins, especially to predict vulnerable epitopes [9]. Accordingly, we applied structure-based network analysis [43] to estimate the topological importance of the Spike trimer (see V C).

We compared regions corresponding to the RBD domain, as a common drug target, and the detected binding site (see Figure 5 a,b,c). We observed that the detected binding site is endowed lower mutation tolerance, as compared to RBD (0.35 vs. 0.22 for the median topological importance). On the other hand, we analyzed the amino acid sequence conservation profiles of the Spike proteins from the coronavirus family (see VB and Supplementary Information for the constructed multiple sequence alignments). We calculated the Valdar's conservation scores [45] and observed that the detected binding site is more conserved amongst the coronavirus family, as compared to the RBD domain, as well as for the set of exposed amino acid residues of Spike (see Figure 5d,e,f). Altogether, these results demonstrate that the detected binding site indeed corresponds to the vulnerable region in the Spike structure of the coronavirus family and indicates a larger applicability domain for drugs targeting it than those targeting RBD.

To summarize, we presented a computational pipeline for rational drug target binding site identification in viral proteins. We applied the pipeline to the SARS-CoV-2 

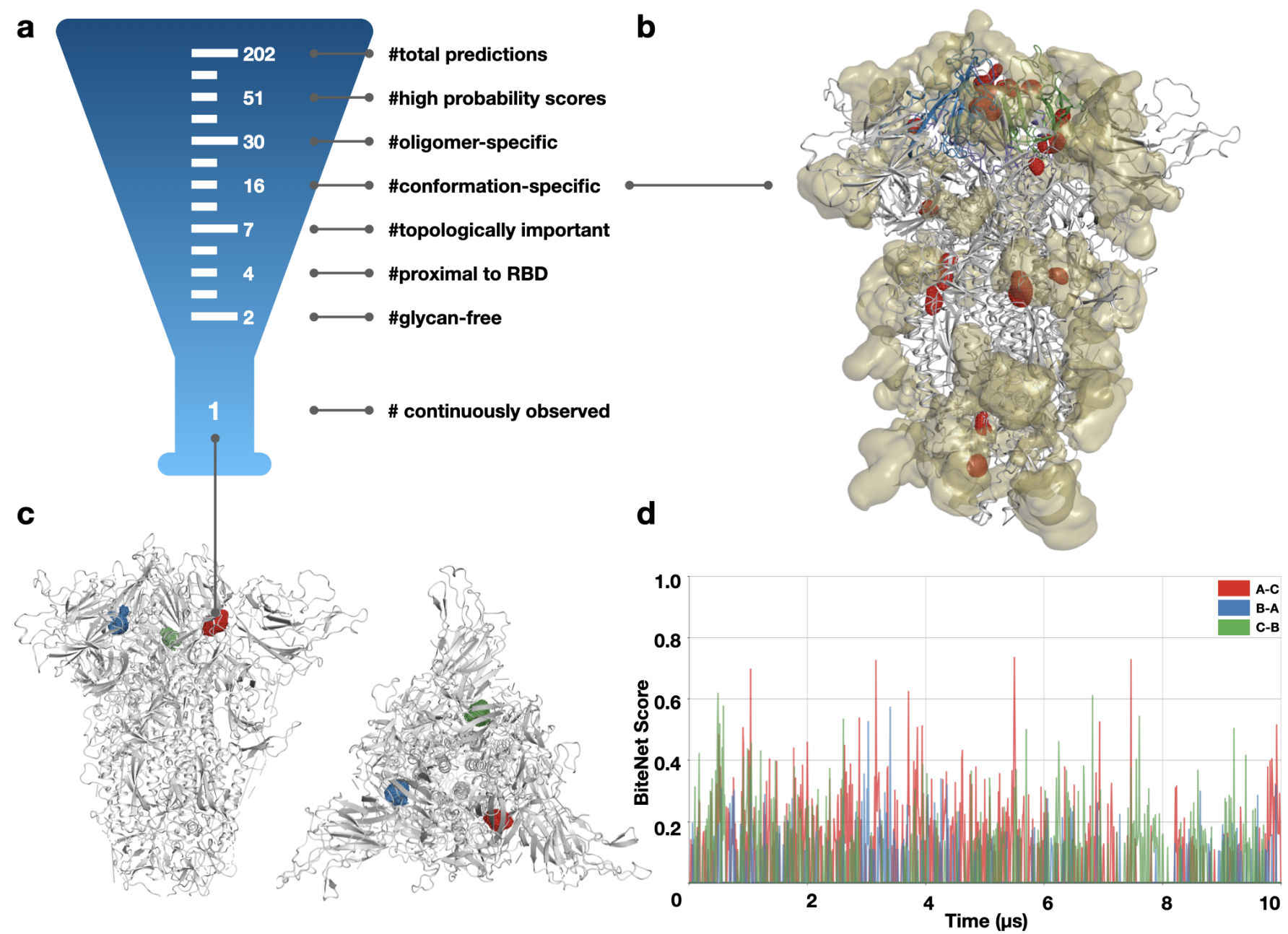

FIG. 1. Target binding site identification pipeline (a) Filtering steps to select the final candidate from the putative binding site predictions (a) Top 16 binding sites predicted in the Spike structure. The predictions are shown with red contours; the glycan densities are shown with yellow transparent surfaces. (c) Side- and top-view on the closed Spike conformation and the three binding sites corresponding to the three pairs of interacting subunits shown with red, green, and blue contours. (d) BiteNet probability scores obtained for the selected binding sites along the $10 \mu \mathrm{s}$ MD trajectory.

Spike Glycoprotein S and identified the vulnerable region, which is more conserved and topologically important than the RBD domain. Molecular docking and molecular dynamics simulations revealed four compounds that stabilize RBD in the closed state, indicating the possibility to inhibit RBD-PD interactions allosterically, hence, abolishing viral activity. Interestingly, one of the compounds, curcumin, possesses anti-inflammatory and antiviral activity [25] and was proved to be effective against SARS-CoV [4]. Unfortunately, we did not find experimental evidence regarding curcumin effectiveness against SARS-CoV-2 or its interactions with Spike, but if curcumin or its analogs have a therapeutic effect, our hypothesis could describe its possible mechanism of action. To conclude, the presented approach would help reduce the high rate of false-positive target binding sites and prepare us better for the next pandemics.

\section{CONCLUSION}

Here we presented computational approach to identify vulnerable regions in viral proteins and applied it to the SARS-CoV-2 Spike Glycoprotein S. By analyzing molecular dynamics trajectories we detected conformationand oligomer-specific glycan-free binding site comprising topologically important amino acid residues and proximal to the receptor binding domain. We confirmed druggability of the binding site by molecular docking of druglike compounds. We observed reduced flexibility of the receptor binding domain in the short molecular dynamics simulations of Spike in complex with four drug-like molecules as compared to the ligand-free simulations. We hypothesized that ligands bound to the detected binding site could lock Spike in the closed state conformation, preventing viral association with the host cell. Overall, 

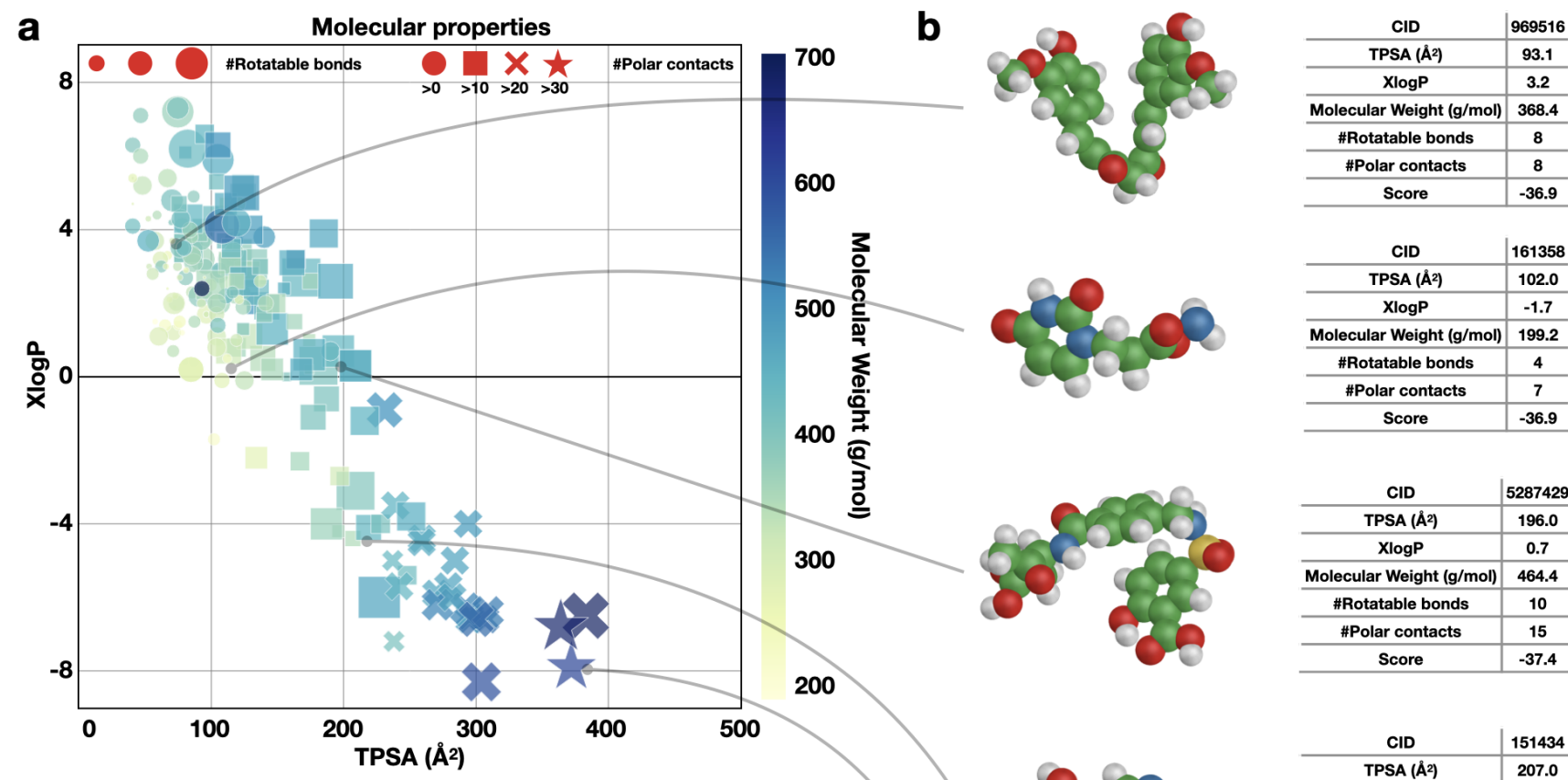

9516

a
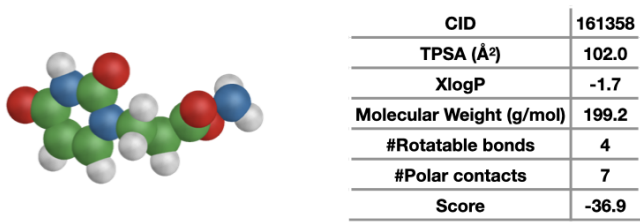

C
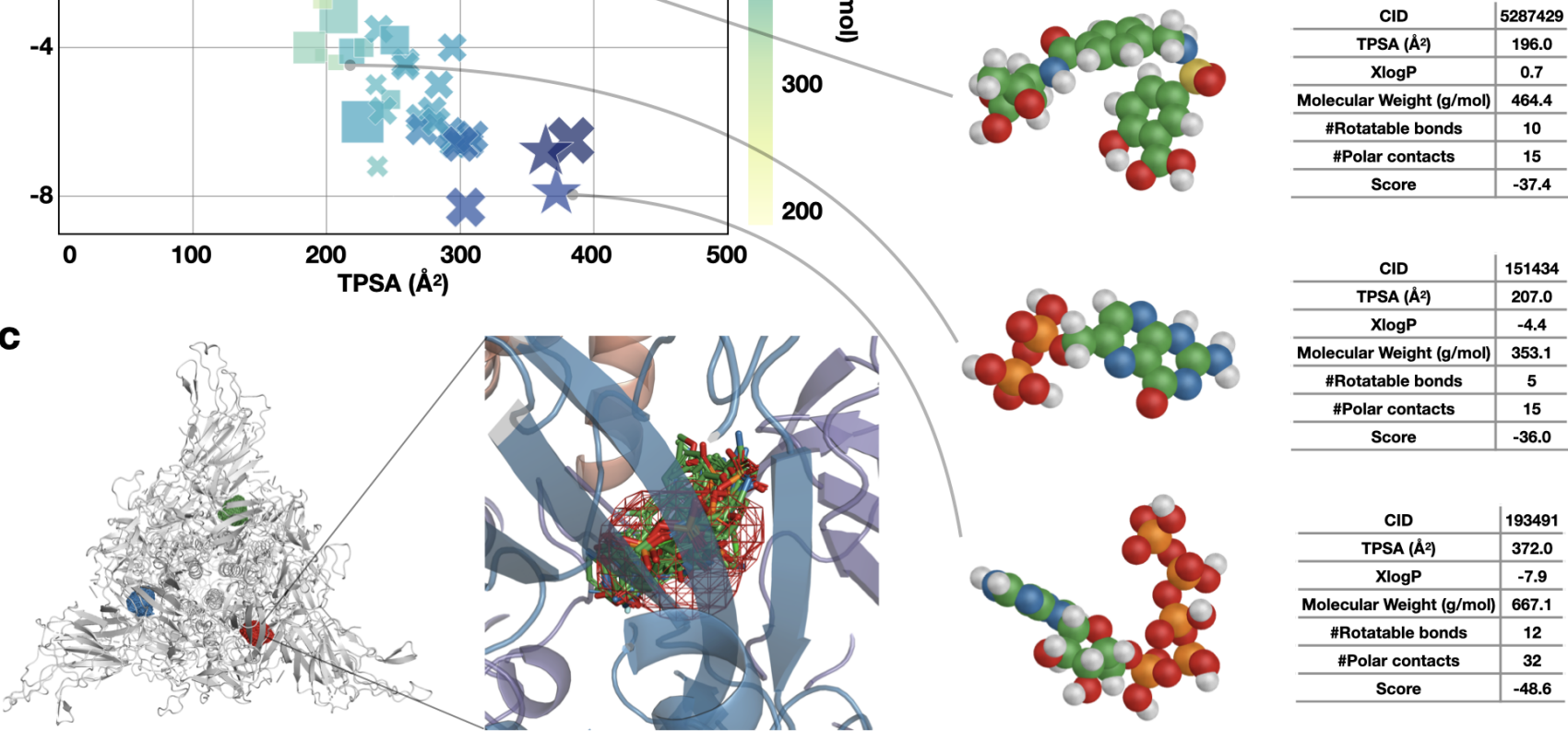

\begin{tabular}{c|c} 
CID & 151434 \\
\hline TPSA $\left(\AA^{2}\right)$ & 207.0 \\
\hline XlogP & -4.4 \\
\hline Molecular Weight (g/mol) & 353.1 \\
\hline \#Rotatable bonds & 5 \\
\hline \#Polar contacts & 15 \\
\hline Score & -36.0
\end{tabular}

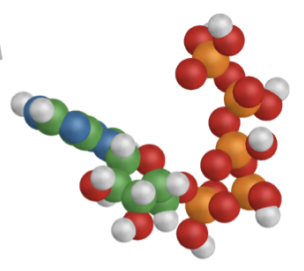

\begin{tabular}{c|c} 
CID & 193491 \\
\hline TPSA (令) & 372.0 \\
\hline XlogP & -7.9 \\
\hline Molecular Weight (g/mol) & 667.1 \\
\hline \#Rotatable bonds & 12 \\
\hline \#Polar contacts & 32 \\
\hline Score & -48.6 \\
\hline
\end{tabular}

FIG. 2. Drug-like molecules identified from molecular docking (a) Distribution of molecular properties for 209 top-scored compounds, including topological polar surface area (TPSA), predicted octanol-water partition coefficient (XlogP), molecular weight, number of rotatable bonds, and number of potential polar contacnts, that is a sum of number of hydrogen bond donors and acceptors. (b) Examples of five different compounds sampled from the distribution; three-dimensional conformers alongside its molecular properties. (c) Superimposed docking poses for the 20 selected compounds.

the presented computational approach could help to better prepare for the next pandemics by identifying the most relevant viral drug target binding sites for drug discovery and design.

\section{METHODS}

\section{A. Binding site identification}

To predict a vulnerable region in Spike, we considered $10 \mu s$ molecular dynamics trajectories of the closed- and pre-fusion Spike states made by D.E.Shaw Research [17. We used the GROMACS trjconv utility to split trajectory into a set of .pdb files [1] that contains only protein chains. Then we applied BiteNet 36 to the closed-state MD simulation using the probability score threshold of
0.01 resulting in 202 putative binding site candidates. This was followed by filtering predictions using the clustering score threshold of 0.01 , leading to 51 predictions. On the next step, we filtered out only binding sites that are within $8 \AA$ from only one protein chain yielding 30 candidates. This was followed by filtering predictions also observed in the prefusion-state MD simulation; thus, we obtained top 16 binding sites. Then, we calculated topological importance for each residue (see VC), and took seven binding sites with the median topological importance higher as compared to the entire Spike. Next, we considered binding sites within $8 \AA$ from the RBD region starting from 350th amino acid residue, resulting in four binding sites. Two predictions were sheltered by glycans and were filtered out; the densities of glycans were calculated for all conformations of the closed-state Spike MD trajectory using VMD [14. Finally, we com- 

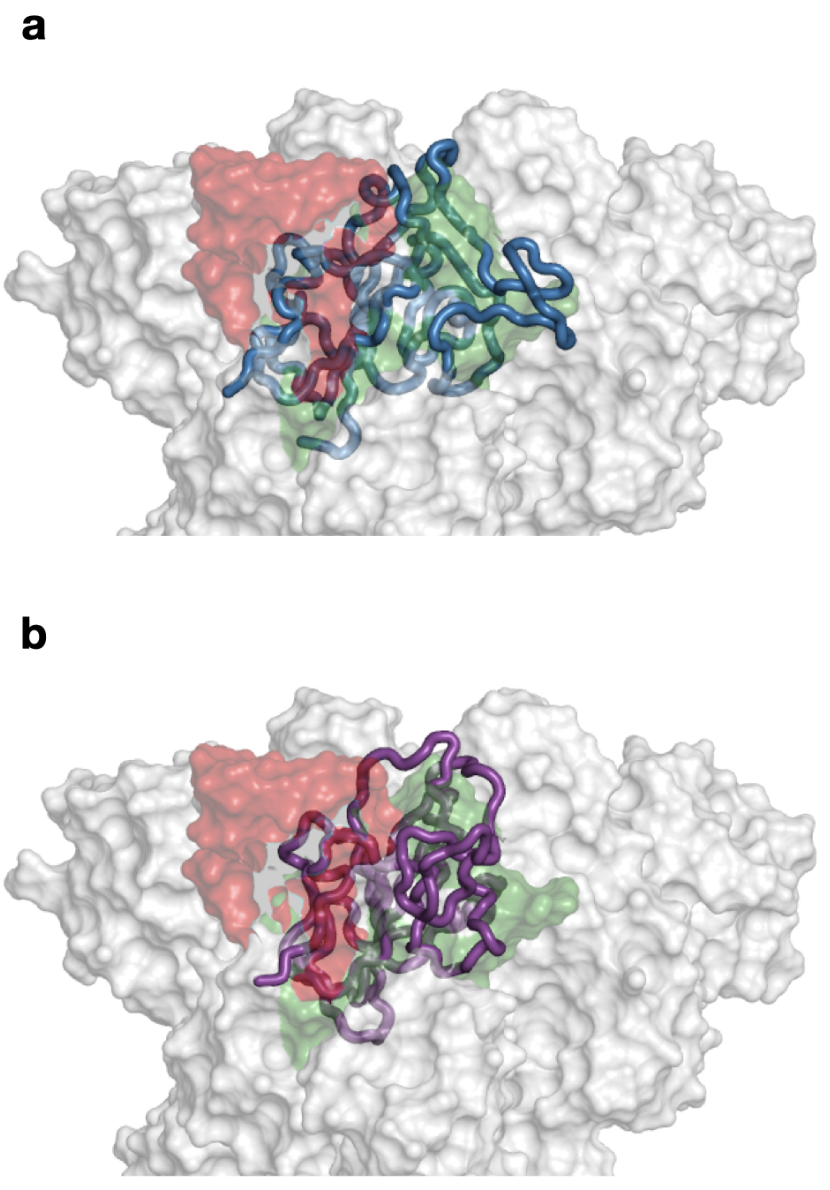
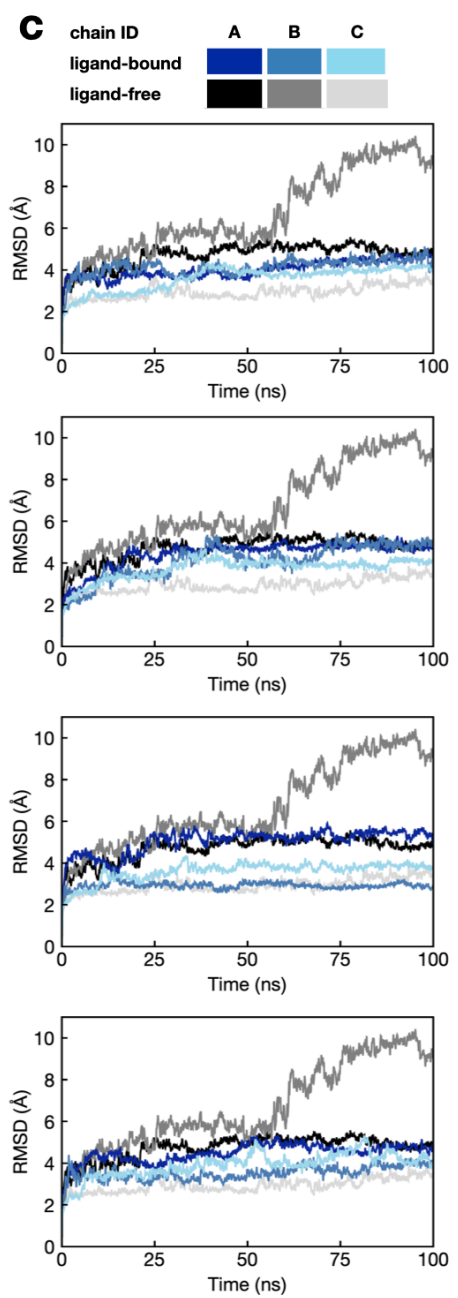

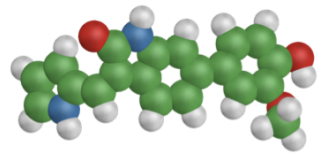

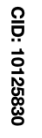
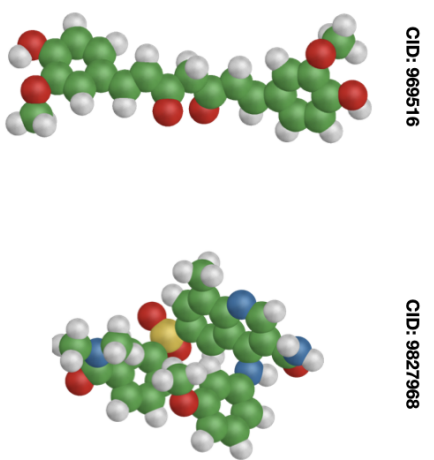

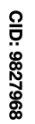

d

FIG. 3. Stabilized RBD in molecular dynamics simulations of ligand-bound Spike structure. (a), (b) The $\mathrm{RBD}$ domain structures corresponding to the last frame of the ligand-bound (blue ribbons) and ligand-free (magenta ribbons) simulations. The closed-state (PDB ID: 6VXX) and the open-state (PDB ID: 6VYB) structures are shown as green and red surfaces, respectively. (c) RMSD profiles of the RBD domains with respect to the initial structure for the ligand-bound (blue scale) and ligand-free (gray scale) simulations for 4 the most stabilizing compounds. (d) Three-dimensional conformers for 4 the most stabilizing compounds.

pared the time ratio, a binding site was observed in the MD simulation, and selected the one corresponding to a higher fraction as the final candidate ( $52 \%$ vs. $5 \%)$.

\section{B. Amino acid conservation analysis}

We retrieved amino acid sequences corresponding to the Spike protein from the InterPro database [28]. To avoid redundancy, we clustered the sequences using the $c d-k i t$ [13] utility with the sequence identity threshold of 0.98 [35] and kept only one entry from each cluster. This procedure resulted in 109 different amino acid sequences of the Spike protein. Then we aligned Spike sequences from the PDB structure and Uniprot (P0DTC2) [5] and constructed multiple sequence alignment (MSA) using mafft 21] based on the obtained pairwise alignment with the --keeplength parameter. Finally, we excluded $\mathrm{PDB}$ sequence from the obtained MSA and calculated the Valdar's conservation score 45] for each amino acid residue position.

\section{Topological importance analysis}

The topological importance was calculated using the structure-based residue interaction network approach [9]. We used the starting frame of the closed-state MD trajectory for the analysis. We disregarded glycans and water molecules beyond $3 \AA$ from the protein chains. We skipped the first steps of the network workflow aimed to add hydrogen atoms 43. The atom names for hydrogen 

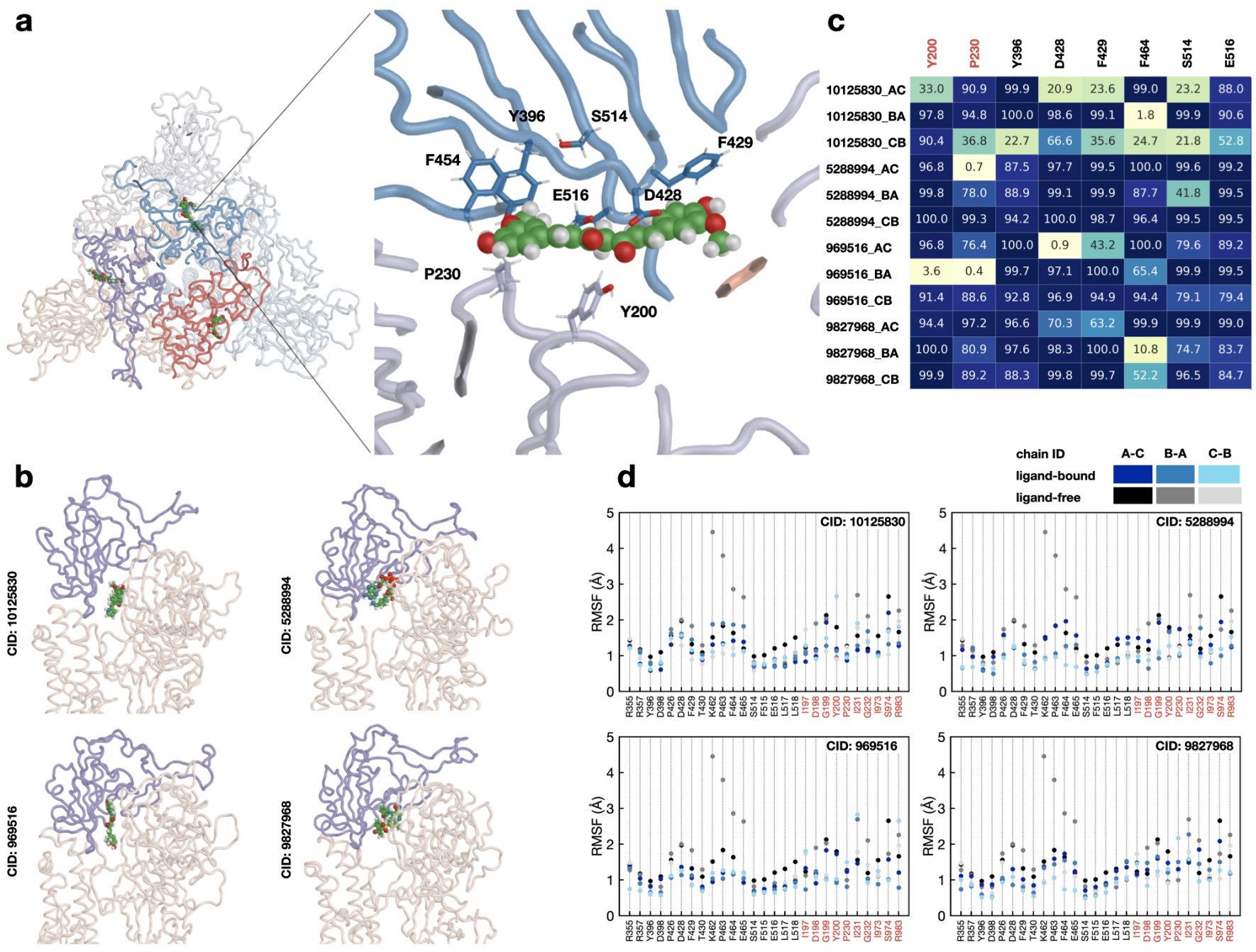

FIG. 4. Protein-ligand interactions (a) Protein-ligand contacts formed within the detected binding site with the CID 969516. Spike is shown with ribbons colored with respect to the chain ID, and the compound is shown with spheres. (b) Interaction interfaces for the top four compounds (one interface per compound) corresponding to the last frames of the MD simulations. (c) The percentage of time the most stable contacts were within $4.0 \AA$ from the ligand during the MD simulations for each interaction interface. (d) RMSF profiles for the amino acid residues observed within $4.0 \AA$ from the ligand during the MD simulations.

and water molecule oxygen were renamed according to the network workflow.

\section{Molecular Docking}

We retrieved investigational, experimental, and approved drug molecules from the DrugBank database [49] in the SMILES format and applied the standardization procedure according to the Chembl structure standardization pipeline [2] yielding 8282 compounds. We want to note that we refer to the PubChem compounds as to the standardized versions of these compounds; however, one should keep in mind that the standardization may change a molecule, particularly stereoisomerization. Then we generated three-dimensional conformations and assign partial charges for each compound using the semiempirical PM3 optimization method implemented in the ORCA software package 30. This procedure yielded 8096 3D conformers, which we converted to the MOL2 format for molecular docking. As the protein structure, we used the Spike conformation from the $10 \mu s$ MD trajectory corresponding to the highest probability score of the binding site of interest. We pre-processed the structure by optimizing side-chain rotamers using Monte Carlo optimization and the MMFF-94 force field. Rectangular box enclosing amino acid residues within $8 \AA$ of the binding cite center with an additional $8 \AA$ margin was used as the sampling space for molecular docking. Protein structure was presented as smoothened grid potentials, while the docking simulations sampled ligand conformations in the internal coordinate space using biased probability Monte Carlo optimization [44] implemented 

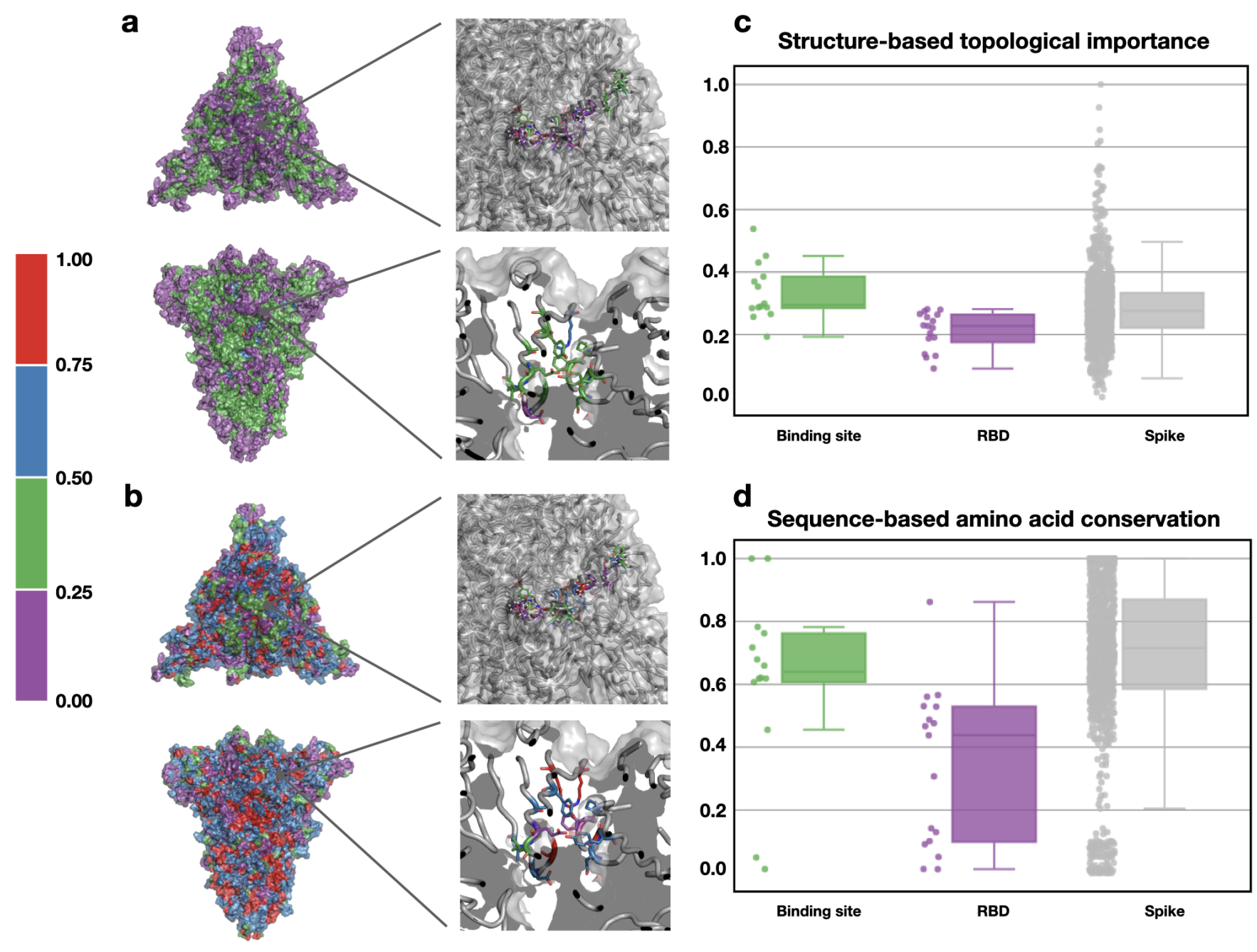

FIG. 5. Structure-based topological importance and sequence-based conservation of the binding site (a), (b) Top- and side-view of the Spike structure colored with respect to the structure-based topological importance of amino acid residues. (c) Box-plots of the structure-based topological importance calculated for the binding site, RBD, and exposed amino acid residues of Spike. (d), (e) Top- and side-view of the Spike structure colored with respect to the Valdar's conservation score calculated for the Spike protein coronavirus family. (c) Box-plots of the Valdar's conservation score calculated for the binding site, RBD, and exposed amino acid residues of Spike.

in ICM-Pro by MolSoft www.molsoft. com with the sampling parameter (docking effort) set to 30. Finally, we constructed the docking hit list ranked with respect to the docking score and selected top 20 compounds, excluding highly similar and those with multiple PO4 groups, for further analysis.

\section{E. Molecular dynamics simulations and analysis}

MD simulations were performed using the GROMACS 2018.6 software [1]. The starting structure was prepared by merging the CHARMM-GUI glycosylated structure [50] with structures by D. E. Shaw Research [17]. The parameters were taken from CHARMM-GUI [50] and reduced to atoms present in structures provided by $\mathrm{D}$.
E. Shaw Research [17]. The detailed procedure to prepare the topology and structural files are described elsewhere [27]. The starting coordinates of the spike protein were pulled to the coordinates of the Spike conformation used for molecular docking by applying position restraints on spike backbone atoms with force constant $10000 \mathrm{~kJ} / \mathrm{mol} / \mathrm{nm}^{2}$ for $1 \mathrm{~ns}$. This resulted in a glycosylated structure with backbone atoms RMSD of $<1 \AA$ from the structure used for molecular docking. The MD parameters for 20 the selected compounds were obtained from Swissparam [59]. The compounds were placed into the binding sites at all three interaction interfaces. For that, the compound positions from the molecular docking were aligned with respect to the other interfaces using MDAnalysis [26]. Overall, 21 systems were prepared, including one ligand-free and 20 ligand-bound. For all 
the systems, periodic boundary conditions were applied. Constant temperature of $303 \mathrm{~K}$ and pressure of 1 bar were maintained by using Nose-Hoover thermostat 31] with time constant of 1.0 ps and isotropic ParrinelloRahman barostat [34] with time constant of 5.0 ps. The Leanard-Jones cutoff was set to $1.2 \mathrm{~nm}$, and LennardJones interactions were smoothly switched to zero at distances higher that $1.0 \mathrm{~nm}$. Electrostatic interactions were treated with the particle mesh Ewald method. A leapfrog integrator was used with an integration step of $2 \mathrm{fs}$. The bond distances and bond angles of water molecules were constrained using the SETTLE algorithm [29], all other bond distances were constrained using the LINCS algorithm [12. Prior to all simulation runs, the potential energy were first minimized using the steepest descent method, followed by 125 ps equllibration MD runs. Production runs for $100 \mathrm{~ns}$ were performed, and frames were saved every 100 ps.

\section{F. Visualization}

We used PyMol v.2.3.5 39] to produce structural images, matplotlib v.3.3.0 [15] and plotly v.4.12.0 [16] python libraries, and the Mathematica v.10.1 package [18] to produce data plots.

\section{ACKNOWLEDGEMENT}

We acknowledge Vladimir Mironov for the structural and topology files used to prepare molecular dynamics simulations. We thank the CSC-IT Center for Science (Espoo,Finland) for computational resources https:// research.csc.fi/-/puhti. We acknowledge Maxim Fedorov and HPC team of CDISE for support our work on the Zhores supercomputer [55]. P.B. was supported by the Academy of Finland (Grant 311031).

\section{SUPPLEMENTARY MATERIALS}

- SupplementaryFile1: List of top 16 binding sites predicted with BiteNet

- SupplementaryFile2: List of top 209 docked druglike molecules along with molecular properties

- SupplementaryFile3: RBD RMSD profiles for the selected 20 drug-like molecules

- SupplementaryFile4: RBD and Binding site RMSF profiles for the selected 20 drug-like molecules

- SupplementaryFile5: MSA for the Spike protein

- SupplementaryFile6: Topological importance and amino acid conservation calculated for the Spike protein
[1] Mark James Abraham, Teemu Murtola, Roland Schulz, Szilárd Páll, Jeremy C. Smith, Berk Hess, and Erik Lindahl. Gromacs: High performance molecular simulations through multi-level parallelism from laptops to supercomputers. SoftwareX, 1-2:19 - 25, 2015.

[2] A Patrícia Bento, Anne Hersey, Eloy Félix, Greg Landrum, Anna Gaulton, Francis Atkinson, Louisa J Bellis, Marleen De Veij, and Andrew R Leach. An open source chemical structure curation pipeline using rdkit. Journal of Cheminformatics, 12(1):1-16, 2020.

[3] Neal K Broomhead and Mahmoud E Soliman. Can we rely on computational predictions to correctly identify ligand binding sites on novel protein drug targets? assessment of binding site prediction methods and a protocol for validation of predicted binding sites. Cell biochemistry and biophysics, 75(1):15-23, 2017.

[4] Longxing Cao, Inna Goreshnik, Brian Coventry, James Brett Case, Lauren Miller, Lisa Kozodoy, Rita E Chen, Lauren Carter, Alexandra C Walls, Young-Jun Park, et al. De novo design of picomolar sars-cov-2 miniprotein inhibitors. Science, 370(6515):426-431, 2020.

[5] UniProt Consortium. Uniprot: a worldwide hub of protein knowledge. Nucleic acids research, 47(D1):D506D515, 2019.

[6] Luisa Di Paola, Hamid Hadi-Alijanvand, Xingyu Song, Guang $\mathrm{Hu}$, and Alessandro Giuliani. The discovery of a putative allosteric site in the sars-cov-2 spike protein using an integrated structural/dynamic approach. Journal of proteome research, 2020.

[7] Elliot D Drew and Robert W Janes. Identification of a druggable binding pocket in the spike protein reveals a key site for existing drugs potentially capable of combating covid-19 infectivity. BMC molecular and cell biology, 21(1):1-13, 2020.

[8] Aled Edwards. What are the odds of finding a covid-19 drug from a lab repurposing screen? Journal of Chemical Information and Modeling, 2020.

[9] Gaurav D Gaiha, Elizabeth J Rossin, Jonathan Urbach, Christian Landeros, David R Collins, Chioma Nwonu, Itai Muzhingi, Olivia M Waring, Alicja PiechockaTrocha, Michael Waring, et al. Structural topology defines protective $\mathrm{cd} 8+\mathrm{t}$ cell epitopes in the hiv proteome. Science, 364(6439):480-484, 2019.

[10] Mahdi Ghorbani, Bernard R Brooks, and Jeffery B Klauda. Critical sequence hot-spots for binding of ncov2019 to ace 2 as evaluated by molecular simulations. bioRxiv, 2020.

[11] Yanxiao Han and Petr Král. Computational design of ace2-based peptide inhibitors of sars-cov-2. ACS nano, 14(4):5143-5147, 2020.

[12] Berk Hess, Henk Bekker, Herman JC Berendsen, and Johannes GEM Fraaije. Lincs: a linear constraint solver for 
molecular simulations. Journal of computational chemistry, 18(12):1463-1472, 1997.

[13] Ying Huang, Beifang Niu, Ying Gao, Limin Fu, and Weizhong Li. CD-HIT Suite: A web server for clustering and comparing biological sequences. Bioinformatics, 26(5):680-682, jan 2010.

[14] William Humphrey, Andrew Dalke, and Klaus Schulten. Vmd: Visual molecular dynamics. Journal of Molecular Graphics, 14(1):33 - 38, 1996.

[15] J. D. Hunter. Matplotlib: A 2d graphics environment. Computing in Science \& Engineering, 9(3):90-95, 2007.

[16] Plotly Technologies Inc. Collaborative data science, 2015.

[17] Plotly Technologies Inc. Molecular dynamics simulations related to sars-cov-2, 2020.

[18] Wolfram Research, Inc. Mathematica, Version 10.1. Champaign, IL, 2020.

[19] M Gordon Joyce, Rajeshwer S Sankhala, Wei-Hung Chen, Misook Choe, Hongjun Bai, Agnes Hajduczki, Lianying Yan, Spencer L Sterling, Caroline E Peterson, Ethan C Green, et al. A cryptic site of vulnerability on the receptor binding domain of the sars-cov- 2 spike glycoprotein. bioRxiv, 2020.

[20] Umesh Kalathiya, Monikaben Padariya, Marcos Mayordomo, Małgorzata Lisowska, Judith Nicholson, Ashita Singh, Maciej Baginski, Robin Fahraeus, Neil Carragher, Kathryn Ball, et al. Highly conserved homotrimer cavity formed by the sars-cov-2 spike glycoprotein: A novel binding site. Journal of Clinical Medicine, 9(5):1473, 2020.

[21] Kazutaka Katoh and Martin C. Frith. Adding unaligned sequences into an existing alignment using MAFFT and LAST. Bioinformatics, 28(23):3144-3146, dec 2012.

[22] Igor Kozlovskii and Petr Popov. Spatiotemporal identification of druggable binding sites using deep learning. bioRxiv, 2020.

[23] Jeremy M Levin, Tudor I Oprea, Sagie Davidovich, Thomas Clozel, John P Overington, Quentin Vanhaelen, Charles R Cantor, Evelyne Bischof, and Alex Zhavoronkov. Artificial intelligence, drug repurposing and peer review. Nature Biotechnology, 38(10):1127-1131, 2020.

[24] Lihong Liu, Pengfei Wang, Manoj S Nair, Jian Yu, Micah Rapp, Qian Wang, Yang Luo, Jasper F-W Chan, Vincent Sahi, Amir Figueroa, et al. Potent neutralizing antibodies against multiple epitopes on sars-cov-2 spike. Nature, 584(7821):450-456, 2020.

[25] Ziteng Liu and Ying Ying. The inhibitory effect of curcumin on virus-induced cytokine storm and its potential use in the associated severe pneumonia. Frontiers in cell and developmental biology, 8:479, 2020.

[26] Naveen Michaud-Agrawal, Elizabeth J. Denning, Thomas B. Woolf, and Oliver Beckstein. Mdanalysis: A toolkit for the analysis of molecular dynamics simulations. Journal of Computational Chemistry, 32(10):2319-2327, 2011.

[27] Vladimir Mironov. Unpublished: Preparing glycosylated structures of sars-cov-2 and ace 2 receptor for md simulations.

[28] Alex L. Mitchell, Teresa K. Attwood, Patricia C. Babbitt, Matthias Blum, Peer Bork, Alan Bridge, Shoshana D. Brown, Hsin Yu Chang, Sara El-Gebali, Matthew I. Fraser, Julian Gough, David R. Haft, Hongzhan Huang, Ivica Letunic, Rodrigo Lopez, Aurélien Luciani, Fabio Madeira, Aron Marchler-Bauer, Huaiyu Mi, Darren A.
Natale, Marco Necci, Gift Nuka, Christine Orengo, Arun P. Pandurangan, Typhaine Paysan-Lafosse, Sebastien Pesseat, Simon C. Potter, Matloob A. Qureshi, Neil D. Rawlings, Nicole Redaschi, Lorna J. Richardson, Catherine Rivoire, Gustavo A. Salazar, Amaia Sangrador-Vegas, Christian J.A. Sigrist, Ian Sillitoe, Granger G. Sutton, Narmada Thanki, Paul D. Thomas, Silvio C.E. Tosatto, Siew Yit Yong, and Robert D. Finn. InterPro in 2019: Improving coverage, classification and access to protein sequence annotations. Nucleic Acids Research, 47(D1):D351-D360, jan 2019.

[29] Shuichi Miyamoto and Peter A Kollman. Settle: An analytical version of the shake and rattle algorithm for rigid water models. Journal of computational chemistry, 13(8):952-962, 1992.

[30] Frank Neese. Software update: the orca program system, version 4.0. Wiley Interdisciplinary Reviews: Computational Molecular Science, 8(1):e1327, 2018.

[31] Shuichi Nosé. A unified formulation of the constant temperature molecular dynamics methods. The Journal of Chemical Physics, 81(1):511-519, 1984.

[32] John P Overington, Bissan Al-Lazikani, and Andrew L Hopkins. How many drug targets are there? Nature reviews Drug discovery, 5(12):993-996, 2006.

[33] Hongchao Pan, Richard Peto, Quarraisha Abdool Karim, Marissa Alejandria, Ana Maria Henao Restrepo, César Hernández García, Marie Paule Kieny, Reza Malekzadeh, Srinivas Murthy, Marie-Pierre Preziosi, et al. Repurposed antiviral drugs for covid-19; interim who solidarity trial results. medRxiv, 2020.

[34] M. Parrinello and A. Rahman. Polymorphic transitions in single crystals: A new molecular dynamics method. Journal of Applied Physics, 52(12):7182-7190, 1981.

[35] Hershna Patel and Andreas Kukol. Prediction of ligands to universally conserved binding sites of the influenza a virus nuclear export protein. Virology, 537:97-103, 2019.

[36] Petr Popov and Igor Kozlovskii. Spatiotemporal identification of druggable binding sites using deep learning (training dataset and software), September 2020.

[37] Florian Prinz, Thomas Schlange, and Khusru Asadullah. Believe it or not: how much can we rely on published data on potential drug targets? Nature reviews Drug discovery, 10(9):712-712, 2011.

[38] Monica Schenone, Vlado Dančík, Bridget K Wagner, and Paul A Clemons. Target identification and mechanism of action in chemical biology and drug discovery. Nature chemical biology, 9(4):232, 2013.

[39] Schrödinger, LLC. The PyMOL molecular graphics system, version 1.8. November 2015.

[40] Jian Shang, Gang Ye, Ke Shi, Yushun Wan, Chuming Luo, Hideki Aihara, Qibin Geng, Ashley Auerbach, and Fang Li. Structural basis of receptor recognition by SARS-CoV-2. Nature, 581(7807):221-224, May 2020.

[41] Mateusz Sikora, Sören von Bülow, Florian EC Blanc, Michael Gecht, Roberto Covino, and Gerhard Hummer. Map of sars-cov-2 spike epitopes not shielded by glycans. bioRxiv, 2020.

[42] Simon J Teague. Implications of protein flexibility for drug discovery. Nature reviews Drug discovery, 2(7):527$541,2003$.

[43] MIT The Walker Lab @ The Ragon Institute of Harvard, MGH, and Olivia Waring. WalkerLabRagon/NetworkAnalysis: Network Analysis Pipeline, March 2019. 
[44] M Totrov and R Abagyan. Protein-ligand docking as an energy optimization problem. Drug-receptor thermodynamics: Introduction and applications, 1:603-624, 2001.

[45] William S.J. Valdar. Scoring residue conservation. Proteins: Structure, Function and Genetics, 48(2):227-241, aug 2002.

[46] Alexandra C Walls, Young-Jun Park, M Alejandra Tortorici, Abigail Wall, Andrew T McGuire, and David Veesler. Structure, function, and antigenicity of the sarscov-2 spike glycoprotein. Cell, 2020.

[47] Yasunori Watanabe, Joel D Allen, Daniel Wrapp, Jason S McLellan, and Max Crispin. Site-specific glycan analysis of the sars-cov-2 spike. Science, 2020.

[48] Chih-Chun Wen, Yueh-Hsiung Kuo, Jia-Tsrong Jan, PoHuang Liang, Sheng-Yang Wang, Hong-Gi Liu, ChingKuo Lee, Shang-Tzen Chang, Chih-Jung Kuo, ShoeiSheng Lee, et al. Specific plant terpenoids and lignoids possess potent antiviral activities against severe acute respiratory syndrome coronavirus. Journal of medicinal chemistry, 50(17):4087-4095, 2007.

[49] David S Wishart, Yannick D Feunang, An C Guo, Elvis J Lo, Ana Marcu, Jason R Grant, Tanvir Sajed, Daniel Johnson, Carin Li, Zinat Sayeeda, et al. Drugbank 5.0: a major update to the drugbank database for 2018. Nucleic acids research, 46(D1):D1074-D1082, 2018.

[50] Hyeonuk Woo, Sang-Jun Park, Yeol Kyo Choi, Taeyong Park, Maham Tanveer, Yiwei Cao, Nathan R. Kern, Jumin Lee, Min Sun Yeom, Tristan I. Croll, Chaok Seok, and Wonpil Im. Developing a fully glycosylated full-length sars-cov-2 spike protein model in a viral membrane. The Journal of Physical Chemistry B, 124(33):7128-7137, 2020. PMID: 32559081.

[51] Daniel Wrapp, Nianshuang Wang, Kizzmekia S Corbett, Jory A Goldsmith, Ching-Lin Hsieh, Olubukola Abiona, Barney S Graham, and Jason S McLellan. Cryo-em structure of the 2019-ncov spike in the prefusion conformation. Science, 367(6483):1260-1263, 2020.

[52] Renhong Yan, Yuanyuan Zhang, Yaning Li, Lu Xia, Yingying Guo, and Qiang Zhou. Structural basis for the recognition of sars-cov-2 by full-length human ace2. Science, 367(6485):1444-1448, 2020.

[53] Renhong Yan, Yuanyuan Zhang, Yaning Li, Lu Xia, Yingying Guo, and Qiang Zhou. Structural basis for the recognition of sars-cov-2 by full-length human ace2. Science, 367(6485):1444-1448, 2020.

[54] Meng Yuan, Nicholas C Wu, Xueyong Zhu, ChangChun D Lee, Ray TY So, Huibin Lv, Chris KP Mok, and Ian A Wilson. A highly conserved cryptic epitope in the receptor binding domains of sars-cov-2 and sars-cov. Science, 368(6491):630-633, 2020.

[55] Igor Zacharov, Rinat Arslanov, Maksim Gunin, Daniil Stefonishin, Andrey Bykov, Sergey Pavlov, Oleg Panarin, Anton Maliutin, Sergey Rykovanov, and Maxim Fedorov. "zhores"-petaflops supercomputer for data-driven modeling, machine learning and artificial intelligence installed in skolkovo institute of science and technology. Open Engineering, 9(1):512-520, 2019.

[56] Peng Zhao, Jeremy L Praissman, Oliver C Grant, Yongfei Cai, Tianshu Xiao, Katelyn E Rosenbalm, Kazuhiro Aoki, Benjamin P Kellman, Robert Bridger, Dan H Barouch, et al. Virus-receptor interactions of glycosylated sars-cov-2 spike and human ace 2 receptor. Cell host Es microbe, 28(4):586-601, 2020.

[57] Na Zhu, Dingyu Zhang, Wenling Wang, Xingwang Li, Bo Yang, Jingdong Song, Xiang Zhao, Baoying Huang, Weifeng Shi, Roujian Lu, et al. A novel coronavirus from patients with pneumonia in china, 2019. New England Journal of Medicine, 2020.

[58] Maxwell I Zimmerman, Justin R Porter, Michael D Ward, Sukrit Singh, Neha Vithani, Artur Meller, Upasana L Mallimadugula, Catherine E Kuhn, Jonathan H Borowsky, Rafal P Wiewiora, et al. Sars-cov-2 simulations go exascale to capture spike opening and reveal cryptic pockets across the proteome. bioRxiv, 2020.

[59] Vincent Zoete, Michel A. Cuendet, Aurélien Grosdidier, and Olivier Michielin. Swissparam: A fast force field generation tool for small organic molecules. Journal of Computational Chemistry, 32(11):2359-2368, 2011. 